\title{
Search for neutrinoless double-beta decay with SuperNEMO
}

\author{
Carla Macolino* on behalf of the SuperNEMO collaboration \\ Laboratoire de l'Accélérateur Lineaire \\ E-mail: macolino@lal.in2p3.fr
}

The SuperNEMO demonstrator is located in the Laboratoire Souterrain de Modane (France) and searches for neutrinoless double beta decay of ${ }^{82} \mathrm{Se}$. Its detection technique, based on tracking and calorimeter measurements, allows the reconstruction of the full kinematics of detected particles, including individual energies and emission angle, resulting in a strong reduction of the background and the possibility to disentangle different $0 v \beta \beta$ decay mechanisms. The goal of the demonstrator is to validate the technique and to reach a sensitivity on the $0 v \beta \beta$ decay half-life of about $\mathrm{T}_{1 / 2}^{0 v}>6 \times 10^{24} \mathrm{yr}$ with "zero background" in the region of interest.

The European Physical Society Conference on High Energy Physics

5-12 July, 2017

Venice

${ }^{*}$ Speaker. 


\section{Introduction}

The SuperNEMO demonstrator searches for neutrinoless double beta decay of ${ }^{82} \mathrm{Se}$ with a technique based on tracking and calorimeter measurements, like its predecessor NEMO3 [1]. This technique allows, in principle, to disentangle different $0 v \beta \beta$ decay mechanisms [2]. In the following sections the main features of the SuperNEMO demonstrator and its installation current status are reported. The sensitivity to $0 v \beta \beta$ decay and the future perspectives of the experiment are also discussed.

\section{The SuperNEMO Demonstrator}

The SuperNEMO detector consists in a planar module $\left(6 \times 4 \times 2 \mathrm{~m}^{3}\right)$ containing, in its center, 7 $\mathrm{kg}$ of ${ }^{82} \mathrm{Se}$ in the form of 36 thin foils of $40-60 \mathrm{mg} / \mathrm{cm}^{2}$ thickness. The source material is composed of ${ }^{82} \mathrm{Se}\left(\mathrm{Q}_{\beta \beta}=2.99 \mathrm{MeV}\right)$ powder mixed with PVA glue arranged on a mylar support frame [3]. On both sides and parallel to the source foil plane a tracker and a calorimeter are installed (see fig. 1). The tracker is a wire chamber operating in Geiger mode with 2034 cells $(40 \mathrm{~mm}$ diameter, $2.7 \mathrm{~m}$ long) and filled with a gas mixture of $\mathrm{He}(95 \%)$, ethanol (4\%) and $\mathrm{Ar}(1 \%)$. The construction and assembly of Geiger cells have been performed in ultraclean conditions. From laboratory measurements dead channels are expected to be less than $1 \%$. The two calorimeter modules are placed parallel to the tracker to precisely measure the deposited energy by the emitted particle. Each calorimeter module is composed of 520 Optical Modules (OMs) made of plastic scintillator (Polystyrene) coupled to 8" and 5" PhotoMultiplier Tubes (PMTs). Thanks to a successfull R\&D effort based on dedicated optical simulations, the energy resolution of the optical modules is on average $8-8.3 \%$ (full width at half maximum) [4] and the time resolution is about $400 \mathrm{ps}$ at $1 \mathrm{MeV}$ (see fig. 2). Trajectories of the electrons emitted from the source foils are measured by the tracker while their curvature can be inferred thanks to a coil producing a $25 \mathrm{G}$ magnetic field (see fig. 1). The energy deposited and the time of flight of the particles are measured by the calorimeter optical modules. Calorimeter calibration is performed with ${ }^{207} \mathrm{Bi}$ sources emitting internal conversion
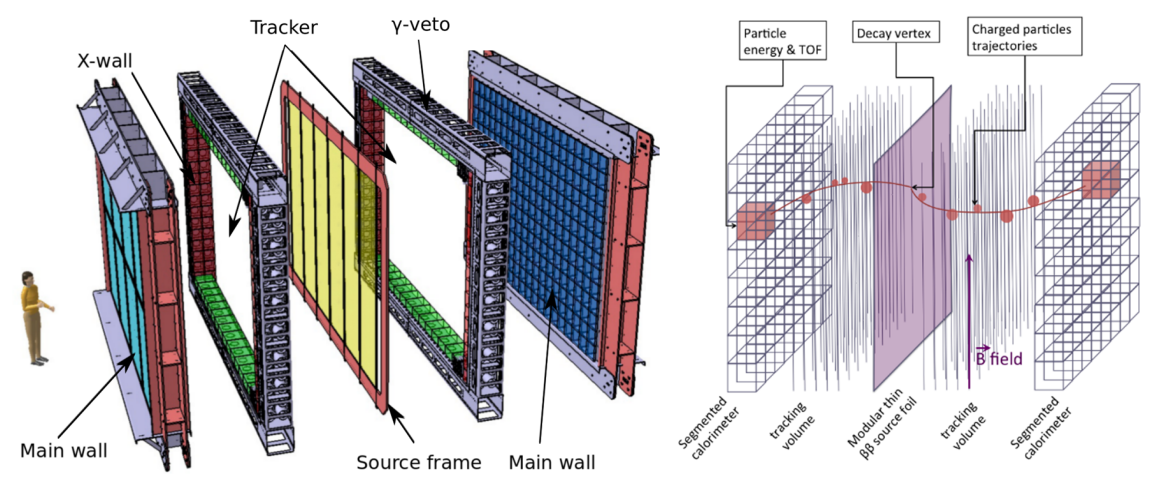

Figure 1: Left: Pictorial view of the SuperNEMO demonstrator. On each side of the source frame a tracker and a calorimeter module are installed. Right: Detection principle of SuperNEMO: trajectories of charged particles emitted from the source foils are measured in the tracker while their energy is deposited into one or more calorimeter modules. 

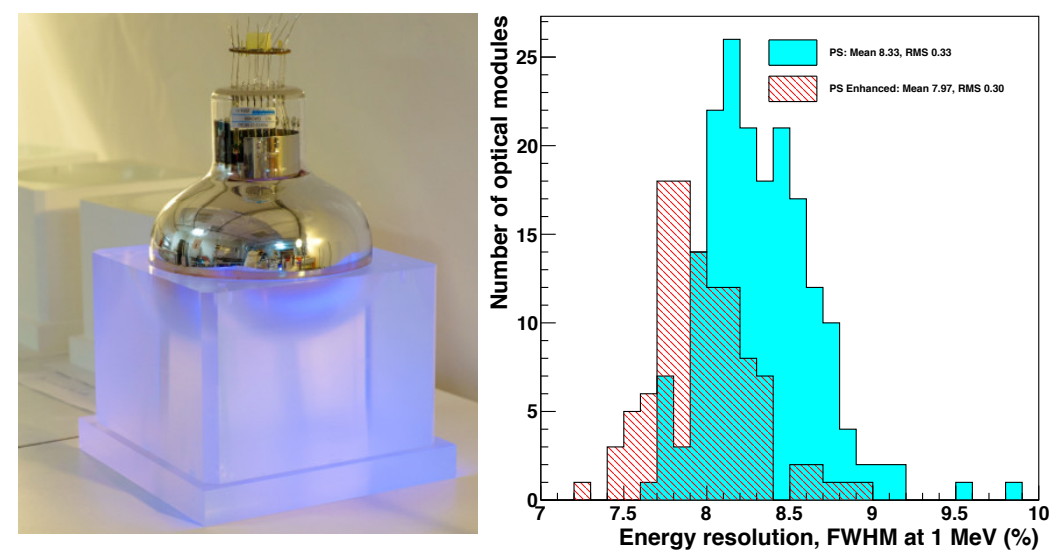

Figure 2: Left: one calorimeter Optical Module (OM) equipped with a 8" PMT. Right: distribution of the energy resolution (FWHM) for the calorimeter Optical Modules. The two different populations refer to normal (light blue) and enhanced (light red) Polystyrene compositions of the scintillators, with an average resolution of $8.3 \%$ and $8 \%$ FWHM, respectively.
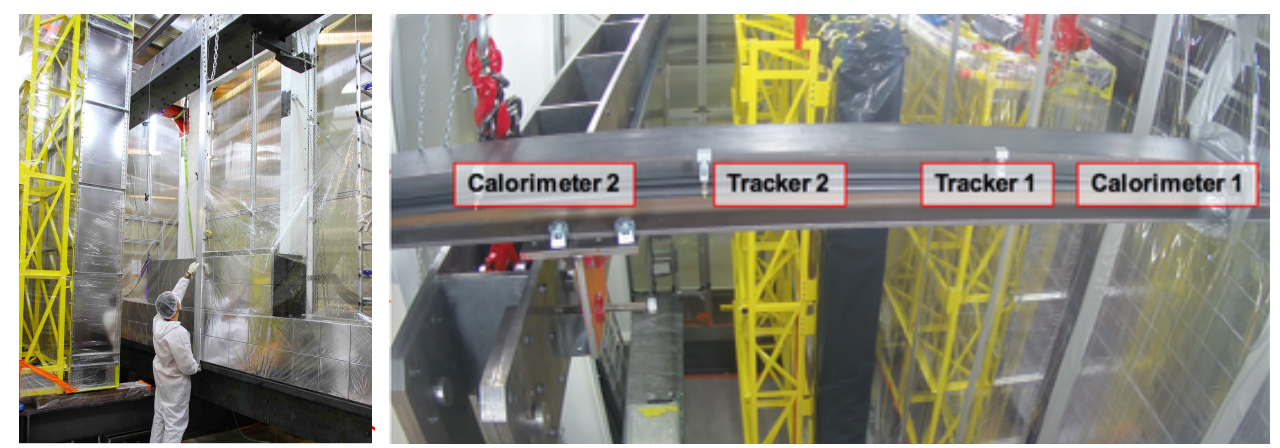

Figure 3: Left: a view of the installation procedure of Optical Modules in the calorimeter wall. Right: Side view of the two calorimeter walls and the two tracking chambers already installed at Modane.

electrons at energies $482 \mathrm{keV}, 976 \mathrm{keV}$, and $1682 \mathrm{keV}$. Calibration sources are inserted in between the source foils through a dedicated system which lowers the sources during calibration runs. The calibration procedure allows energy stability within $1 \%$. At present the SuperNEMO demonstrator is currently under integration at the Laboratoire Souterrain de Modane. Both sides of the tracker and calorimeter have been installed (see fig. 3) and half of the detector is already in its commissioning phase. The source foils and calibration frames will be installed within 2017. Data taking is expected to start by middle of 2018 .

\section{Radiopurity requirements and sensitivity}

In order to reach the target sensitivity, the SuperNEMO demonstrator has established different requirements in terms of radiopurity of its components. In particular the source foils internal contamination must not exceed 2 and $10 \mu \mathrm{Bq} / \mathrm{kg}$ for ${ }^{208} \mathrm{Tl}$ and ${ }^{214} \mathrm{Bi}$, respectively. The gas in the tracking chamber must satisfy Radon $\left({ }^{222} \mathrm{Rn}\right)$ levels below $150 \mu \mathrm{Bq} / \mathrm{m}^{3}$ and the radiopurity of component materials in the vicinity of source foils must be at the levels of few $\mathrm{mBq} / \mathrm{kg}$. 
A dedicated high sensitivity detector called BiPo-3 with a total sensitive area of $3.6 \mathrm{~m}^{2}$ [5] has been built by the SuperNEMO collaboration in 2012 to measure the internal contamination of source foils at the levels of few $\mu \mathrm{Bq} / \mathrm{kg}$ for ${ }^{208} \mathrm{Tl}$ and ${ }^{214} \mathrm{Bi}$ (from the Thorium and Uranium series, respectively). BiPo-3 is operational since 2013 and is located in the Canfranc Underground Laboratory (Spain). It is divided in two modules, each of them hosting 20 pairs of thin polystyrene scintillators coupled to 5 " low background PMTs. The ${ }^{208} \mathrm{Tl}$ contamination can be inferred by detecting the ${ }^{212} \mathrm{BiPo}$ cascade, i.e. an electron from the $\beta$ decay of ${ }^{212} \mathrm{Bi}$ and a delayed $\alpha$ (of 8.8 $\mathrm{MeV}$ energy) from the decay of ${ }^{212} \mathrm{Po}$ (with half-life of $300 \mathrm{~ns}$ ). Preliminary measurements for a subgroup of source foils indicate a contamination in ${ }^{208} \mathrm{Tl}$ between 10 and $30 \mu \mathrm{Bq} / \mathrm{kg}$ at $90 \%$ C.L. A big effort has been made to minimize the ${ }^{222} \mathrm{Rn}$ contamination in SuperNEMO and achieve levels of about $0.15 \mathrm{mBq} / \mathrm{m}^{3}$. Indeed, ${ }^{222} \mathrm{Rn}$ can emanate from detector materials with ${ }^{226} \mathrm{Ra}$ impurities, can diffuse from outside materials or can be injected through the input gas in the tracker. Material selection has been performed to identify the most tight materials with the minimum Rn diffusion coefficient; thanks to this, the use of nylon between the tracker and the calorimeter has been validated [6]. Different radiopure stainless steel emanation chambers have been built by different groups of the collaboration to measure in particular Rn emanation from materials in contact with the tracker gas. A large chamber (with a $0.7 \mathrm{~m}^{3}$ volume) with a $3 \mathrm{mBq}$ sensitivity is able to measure large volume samples or films with surfaces up to $80 \mathrm{~m}^{3}$ [7]. Small setups with sensitivity of 0.2 $\mathrm{mBq}$ and few liters volume have been used for smaller samples.

A Radon Concentration Line (RnCL) has also been developed to measure the final activity of the tracker. The system is composed of an electrostatic Radon detector coupled to a carbon trap cooled to $-50{ }^{\circ} \mathrm{C}$. Nitrogen is flushed inside each quarter of the tracker and then it is directed to the RnCL to estimate the Radon emanation from the tracker internal materials. Performed measurements resulted in few $\mathrm{mBq}$ of Radon activity [8]. Considering a gas flushing rate of $2 \mathrm{~m}^{3} / \mathrm{h}$, Radon levels of $0.15 \mathrm{mBq} / \mathrm{m}^{3}$ in the tracker will be achieved. Finally all the detector materials have also been screened by low-background gamma spectrometry with High Purity Germanium detectors (HPGe). Measured activity levels for ${ }^{40} \mathrm{~K},{ }^{238} \mathrm{U},{ }^{214} \mathrm{Bi}$ and ${ }^{208} \mathrm{Tl}$ range from few $0.1 \mathrm{mBq} / \mathrm{kg}$ to few $100 \mathrm{mBq} / \mathrm{kg}$ [9].

The sensitivity on the half-life of neutrinoless double beta decay is estimated by considering the target levels of background contaminations (Tl, Bi and Rn) and the $2 v \beta \beta$ decay of ${ }^{82} \mathrm{Se}$ with a $8 \%$ (FWHM) energy resolution. With about 0.1 events expected in the region of interest (between 2.8 and $3.2 \mathrm{MeV}$ ) and an exposure of $17.5 \mathrm{~kg} \cdot \mathrm{yr}$, the sensivity on the half-life is $\mathrm{T}_{1 / 2}^{0 v}=5.9 \times 10^{24} \mathrm{yr}$, which translates in a range of the effective neutrino mass between 0.20 and $0.55 \mathrm{eV} \mathrm{[10].}$

\section{Conclusions and future perspectives}

The SuperNEMO demonstrator is currently in the last stages of its installation phase which will be completed in the first months of 2018. After $2.5 \mathrm{yr}$ of data taking, the sensitivity goal of the detector will be reached if the required backgroud levels of internal and external contaminations will be achieved. To enhance the sensitivity and partially explore the inverted region of the effective Majorana neutrino mass (between 40 and $110 \mathrm{meV}$ ), the requirement on the $0 v \beta \beta$ half-life is at the level of $\mathrm{T}_{1 / 2}^{0 v}>10^{26} \mathrm{yr}$. With 20 detector modules based on the design of the demonstrator and 

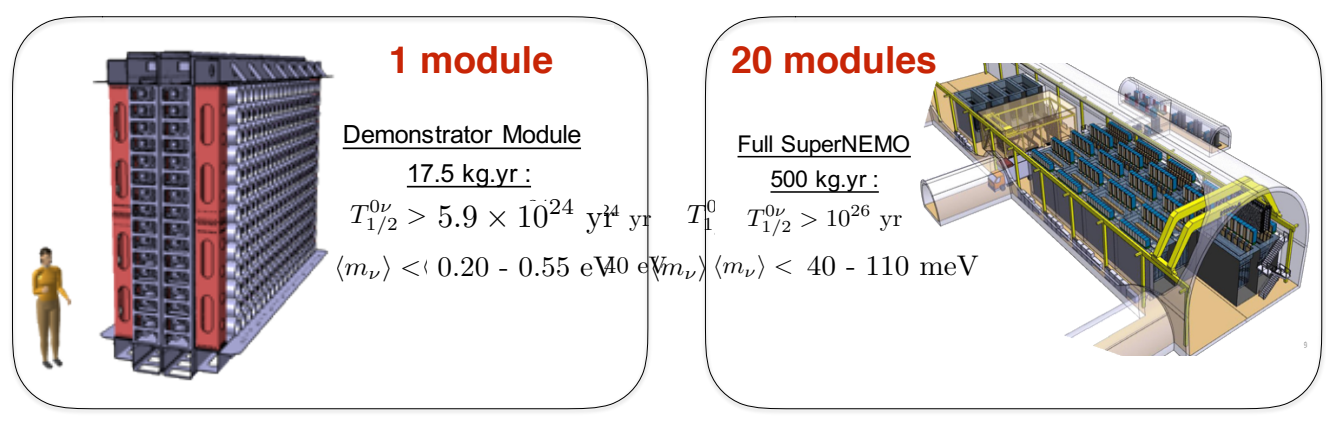

Figure 4: Left: Sensitivity projection of the SuperNEMO Demonstrator. Right: Estimated sensitivity for the complete SuperNEMO detector.

hosting each $5 \mathrm{~kg}$ of ${ }^{82} \mathrm{Se}$, SuperNEMO will be able to fulfill this requirement after an exposure of $500 \mathrm{~kg} \cdot \mathrm{yr}$ (see fig. 4).

\section{References}

[1] R. Arnold et al., Results of the search for neutrinoless double- $\beta$ decay in $100{ }^{100}$ Mo with the NEMO-3 experiment, Phys. Rev. D 92 (2015) 072011

[2] R. Arnold et al., Probing New Physics Models of Neutrinoless Double Beta Decay with SuperNEMO, Eur. Phys. J. C 70 (2010) 927-943

[3] A. Jeremie and A. Remoto, The SuperNEMO $\beta \beta$ source production, 38th International Conference on High Energy Physics (ICHEP 2016), Chicago, USA, August 3-10, 2016

[4] A.S. Barabash et al., Calorimeter development for the SuperNEMO double beta decay experiment, Nucl. Instrum. Meth. A 868 (2017) 98-108

[5] A.S. Barabash et al., The BiPo-3 detector for the measurement of ultra low natural radioactivities of thin materials, JINST 12 (2017) no.06, P06002

[6] F. Mamedov, I. Stekl and K. Smolek, Measurement of the radon diffusion through a nylon foil for different air humidities, Low Radioactivity Techniques 2015 (LRT2015), AIP Conference Proceedings 1672 (2015) 140007

[7] C. Cerna, B. Soulé and F. Perrot, Radon emanation based material measurement and selection for the SuperNEMO double beta decay experiment, Low Radioactivity Techniques 2015 (LRT2015), AIP Conference Proceedings 1672 (2015) 050002

[8] X.R.Liu, Radon mitigation strategy and results for the SuperNEMO experiment, XXVII International Conference on Neutrino Physics and Astrophysics (Neutrino 2016), London, UK, July 4-9, 2016

[9] F. Perrot, Strategy of HPGe screening measurements in the SuperNEMO experiment, Low Radioactivity Techniques 2013 (LRT2013), AIP Conference Proceedings 1549 (2013) 050002

[10] S. Calvez, Ov $\beta \beta$ sensitivity with the SuperNEMO demonstrator, 52nd Rencontres de Moriond EW 2017, La Thuile, Italy, Mars 18-25, 2017 